\title{
Study on Sealing Performance of Oil Seal with Micro-pores Textured in Rotary Shaft Surface
}

\author{
CHENG Xiang-ping*, ZHANG You-liang, KANG Lin-ping \\ Institute of Applied Physics, Jiangxi Academy of Sciences, Nanchang 330029, China)
}

\begin{abstract}
In order to improve the reliability and the long life cycle of the lip seals, comprehensive consideration of the liquid film cavitation and JFO mass conservation boundary conditions, the geometry model of the oil seal with micro-diamonds textured on the shaft surface is given, and its mathematical model is built, the relevant numerical calculation method is used, eventually that the film pressure distribution and the micro diamond pores structure parameters effect on the seal performance are obtained under the different operating conditions. The results showed that the dynamic pressure effect caused by the diamond pores can make the oil film pressure field between the axial surface and the lip produced regular axial and radial wave change. At the same time, the change of working conditions strengthen or weaken the change law of the oil film pressure field, so which make the reliability of the liquid pressure, lubricating property and pump suction effect also change accordingly. The size, shape and pores direction of the microdiamonds texture have great influence on the oil seal performance and lubrication characteristics, which can help to reduce the leakage rate, control the pump suction direction, stable the liquid pressure and reduce the friction. In order to improve the stability of the liquid film and pump suction effect, reduce the leakage rate, friction and wear, the axial surface micro-diamond pores texture of which the cone width ratio is 0.4-0.6, the pores depth is $1.5-4.5 \mu \mathrm{m}$ and the tilt angle is $40^{\circ}-50^{\circ}$ shall be selected for the oil seals.
\end{abstract}

In recent years, with the continuous improvement of many requirements for the lubrication characteristics of the key friction and wear parts of the rotating equipment, the micro-textured technology of the contact surfaces has developed rapidly with its excellent lubrication performance. At present, with the growing expansion of high precision, cutting-edge industry, the requirements of which the environmental protection and energy saving, seal reliability and long life cycle of the types of lip seals are also more and more high, so need to design and develop long use period and better performance of the new oil seals, which become a problem to be solved, lip seals with axial surface micro forming technology have been put on the agenda, and incomparable better performances than traditional oil seals are found, the rotate equipments in agricultural machinery, aerospace, marine, petroleum and petrochemical industries have been gradually put into use, and the effect is excellent. Domestic and foreign scholars[1-7] had also carried out a large number of theoretical and experimental studies to reveal the micro-woven structure of the axial surface and improve the friction lubrication mechanism between contact surfaces, effectively improving the lubrication performance. Moreover, it was found that the shape, size, pores depth, pores orientation and area ratio of axial surface micro-texture[8-14] had greatly influenced on the lubrication performance of the oil seal. In addition, the mechanism of the surface micro-modeling to which improve the lubrication state and the sealing performance is very complex, which is not only dependent on its shape and structure, but also has a great relationship with working conditions, and its internal mechanism needs to be further revealed. In view of this, aimed at the different coaxial surfaces microstructure textures as the research object, which mass conservation Reynolds governing equation is built between sealing surfaces in this paper, and the distribution of film pressure was analyzed, the influence of structural parameters and operating parameters of the micro-model textures on the sealing performance of micro-pores shaft surface was studied, its lubrication mechanism was delved into.

\section{Model}

\subsection{Structure model}

Fig. 1 shows the assembly structure and local profile of typical axial micro-pores texture oil seal, and the micropores can be triangles, squares, rectangles, ellipses, diamonds and circles, etc. Along the axial and circumferential directions, the suitable micro-pores are uniform etched in the axis surfaces. However, there are only two rows of micro-pores in the axial direction

\footnotetext{
* Corresponding author: $654268480 @$ qq.com
} 
between the lip and axial surface, $\mathrm{L}$ is the axial length in sealing area, $h_{0}$ is the balance film thickness, $\mathrm{h} 1$ is the pores depth, $a$ and $b$ are respectively the effective half side length in the calculation formula for the area of micro-pores, the side length of circulars and squares is $a=b=r$, the shape factor of all micro-pores is $\gamma=b / a$ except the circles and squares. The angle between the semi-long axis and the tangent direction of the rotating axis through the pore center is $\alpha_{1}$.

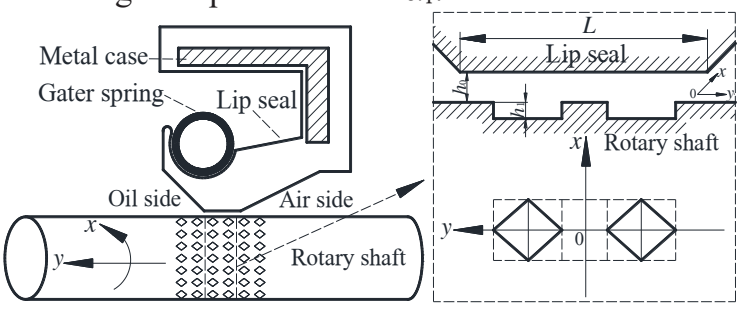

(a) micro-pores distribution of shaft surface

(b) Schematic diagram of micro-pores in a cycle

Fig.1 Schematic of the lip seal structure and local profile

\subsection{Mathematical model}

It is assumed that the sealing medium is Newtonian fluid, and its viscosity and pressure remain constant along the direction of liquid film thickness. In consideration of liquid film cavitation, JFO mass conservation Reynolds control equation is adopted:

$$
\frac{1}{r} \frac{\partial}{\partial \phi}\left(\frac{h^{3}}{12 \mu} \frac{\partial p}{\partial \phi}\right)+\frac{\partial}{\partial r}\left(\frac{r h^{3}}{12 \mu} \frac{\partial p}{\partial r}\right)=\frac{r U}{2} \frac{\partial(\theta h)}{\partial \phi}
$$

Type in the

$\theta=\rho / \rho_{1}\left(\rho\right.$-the medium density, $\rho_{1}$-the medium density in non-cavitation region), in the non-cavitation region: $\theta=1, p>p_{\text {cav }}$, in the cavitation region: $0<\theta<1, p=p_{\text {cav. }} h$ is the liquid film thickness; $\boldsymbol{U}$ is the rotating shaft speed; $p$ and $p_{\text {cav }}$ are respectively oil pressure and cavitation pressure.

Assuming that the lip part and the axial surface are smooth contact surfaces, the liquid film thickness equation of the sealing area is as follows:

$$
h=\left\{\begin{array}{l}
h_{0} \quad \text { pores outside } \\
h_{0}+h_{1} \text { pores inside }
\end{array}\right.
$$

Since the micro-pores are arranged periodically on the rotating shaft surface, the single period is selected as the research object in Fig. 1.

The mandatory boundary condition:

$$
\left\{\begin{array}{l}
p\left(-1 \frac{1}{2}, Y\right)=p_{\mathrm{o}} \\
p\left(\begin{array}{ll}
1 \frac{1}{2}, & Y
\end{array}\right)=p_{\mathrm{a}}=p_{\mathrm{i}} \\
\theta\left(-1 \frac{1}{2}, \quad Y\right)=\theta\left(1 \frac{1}{2}, Y\right)
\end{array}\right.
$$

Periodical boundary conditions:

$$
\left\{\begin{array}{l}
p\left(X,-\frac{1}{2}\right)=p\left(X, \frac{1}{2}\right) \\
\theta\left(X,-\frac{1}{2}\right)=\theta\left(X, \frac{1}{2}\right)
\end{array}\right.
$$

JFO cavitation conditions:

$$
\left\{\begin{array}{l}
p>p_{c}, \theta=1 \\
p<p_{c}, 0<\theta<1
\end{array}\right.
$$

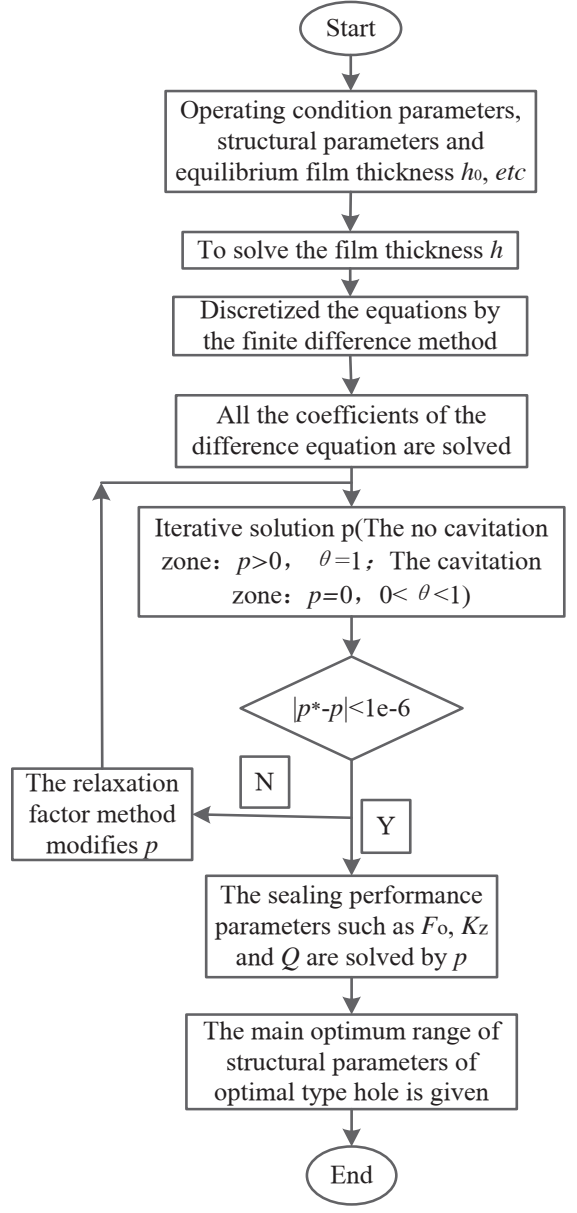

Fig.2 Flow chart for computation

The calculation process is shown in Fig.2, the process is as follows:

(1) Input the base film thickness, geometric and operating parameters;

(2) Combining JFO mass conservation boundary conditions, the finite difference method is used to transform equation (1) into a difference equation by discrete decomposition, and the initial pressure $p_{\mathrm{o}}$ is used to solve all the coefficients of the difference equation.

(3) In the process of iteratively calculating pressure $p$, the relaxation iteration method is used to correctly calculate $p$. After repeated iterations, until the pressure convergence criterion conforms to the given value, the iteration is stopped and the next step is continued.

(4) The finally solved pressure is used to calculate parameters such as the liquid film stiffness, opening force and leakage rate, etc. The specific algorithm is shown in the literature ${ }^{[15]}$.

\section{Calculation results and discussion}

(1) Structural parameters: $L=100 \mu \mathrm{m}, h_{0}=2.0 \mu \mathrm{m}$, $h_{1}=4 \mu \mathrm{m}$, pores number in per cycle $n_{\mathrm{s}}=2, \gamma=0.5$, $a=15 \mu \mathrm{m}, \alpha_{1}=0$.

(2) Operating parameters: the oil side pressure $p$ ${ }_{0}=0.12 \mathrm{MPa}$, air side pressure $p_{\mathrm{a}}=p_{\mathrm{i}}=0.1 \mathrm{MPa}$, cavitation pressure $p_{\mathrm{c}}=0$, shaft rotary speed $U=200 \mathrm{r} / \mathrm{min}$, viscosity of oil $\mu=0.01$ Pa.s. 
When calculating and analyzing the influence of the structure and operation parameters on the sealing performance, the other geometric and the operating parameters remain unchanged, except as specified.

\subsection{Influence of the operating parameters on the sealing performance}

\subsubsection{Impact of po}

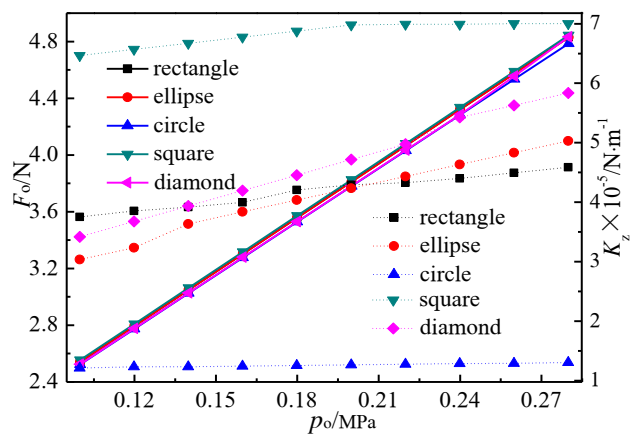

(a) $F_{0} 、 K_{\mathrm{z}} \& p_{0}$

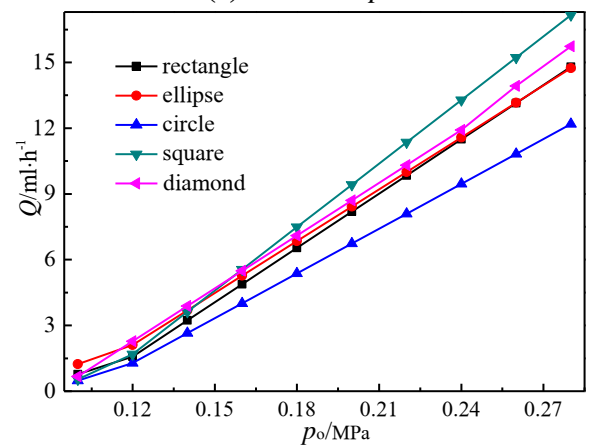

(b) $Q \& p_{0}$

Fig.3 Oil film pressure diagram under the change of $p_{\mathrm{o}}$

It is shown that the influence on the sealing performance of each pore texture in oil seal zone in the Fig. 3 when $p_{\mathrm{o}}$ changes, the solid curve in the figure corresponds to the vertical axis on the left, while the virtual curve corresponds to the vertical axis on the right (Fig.3(a)), the $x$-axis is shared. With the continuous increase of the pressure, it is shown that the $K_{\mathrm{z}}$ and $F_{o}$ of the other pores texture lip seals show an increasing tendency, except that the liquid film stiffness of the circular texture pores tends to be horizontal linear, while the leakage rate of all pores increases constantly with the increase of $p_{0}$, that is, the stability of the liquid film (except the circular pores) and the bearing capacity become larger and larger, and the leakage ratio is becoming more and more big with the increase of $p_{\mathrm{o}}$. This indicates that the static pressure effect is increasing with the pressure difference increasing, and the leakage rate of the all pores also increases with the increase of the oil side pressure, while the dynamic pressure effect of axial surface micro-pores remains unchanged under the constant speed condition, under the combined action of dynamic, static pressure effect and shear effect, the pump drain effect of axial surface pores weakens, resulting in the continuous increase of the leakage rate. It can also be seen that the open force of each pore is almost same under the same pressure. $Q$ is sorted

as $Q_{\text {(square) }}>Q_{\text {(diamond) }}>Q_{\text {(rectangle) }}>Q_{\text {(ellipse) }}>Q_{\text {(circle). When }}$ the oil side pressure is less than $0.14 \mathrm{MPa}, K_{\mathrm{z}}$ is sorted as $K_{\mathrm{z} \text { (square) }}>K_{\mathrm{z} \text { (rectangle) }}>K_{\mathrm{z} \text { (diamond) }}>K_{\mathrm{z} \text { (ellipse) }}>K_{\mathrm{z}} \quad$ (circle). When the scope of $p_{\mathrm{o}}$ is $0.14 \mathrm{MPa}<p_{\mathrm{o}}<0.21 \mathrm{MPa}, K_{\mathrm{z}}$ is sorted as $K_{\mathrm{z} \text { (square) }}>K_{\mathrm{z} \text { (diamond) }}>K_{\mathrm{z} \text { (rectangle) }}>K_{\mathrm{z} \text { (ellipse) }}>K_{\mathrm{z} \text { (circle). }}$ When the $p_{\mathrm{o}}$ is greater than $0.21 \mathrm{MPa}, K_{\mathrm{z}}$ is sorted as $K_{\mathrm{z}}$ (square) $>K_{\mathrm{z} \text { (diamond) }}>K_{\mathrm{z} \text { (ellipse) }}>K_{\mathrm{z} \text { (rectangle) }}>K_{\mathrm{z} \text { (circle) }}$.

\subsubsection{Influence of $U$}

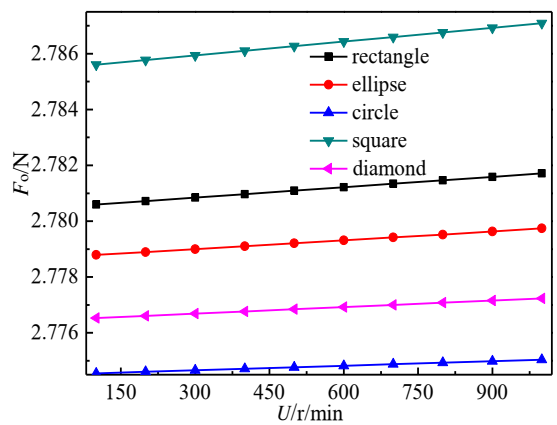

(a) $F_{0} \& U$

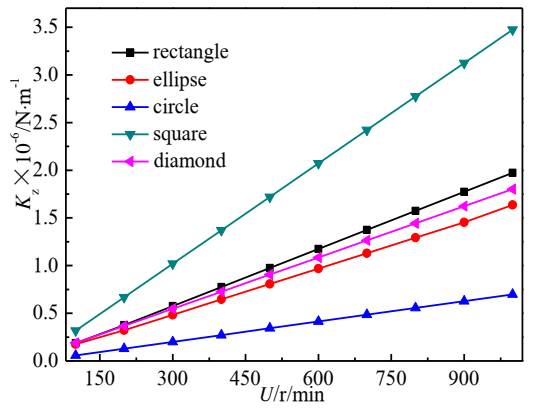

(b) $K_{\mathrm{z}} \& U$

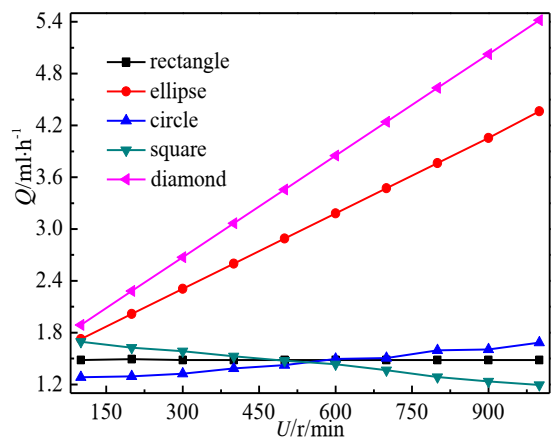

(c) $Q \& U$

Fig.4 Effect of $U$ on sealing performance parameters

It is shown that the influence law of rotary speed on the sealing performance of each pore texture in oil seal zone in fig.4. As the chart shows, $K_{\mathrm{z}}$ and $F_{o}$ of each pore texture show a trend of linear increase with the increase of the rotary speed, and the change range is larger, except for the square, the $Q$ of other pores increases with the increase of rotating speed. The reason is that the hydrodynamic pressure effect and pump drain effect in the sealing area will increase significantly with the increase of the rotary speed, therefore, the bearing capacity and stability of the liquid film are constantly 
strengthened. In addition to the square micro-pores, the $Q$ of the other micro-pores also increases, however, the increase of pump drain effect caused by square micropores with the increase of velocity is stronger than that caused by centrifugal force. Therefore, the media which about to be released from the sealing area will be pumped back to the oil side in large quantities, so $Q$ is going to decrease as speed goes up. It can also be seen that $F_{o}$ and $K_{\mathrm{z}}$ are sorted as follows: $F_{o \text { (square) }}>F_{o \text { (rectangle) }}>F_{o \text { (ellipse) }}>F_{o \text { (diamond) }}>F_{o \text { (circle) }}$ and $K_{\mathrm{z}}$ (square) $>K_{\mathrm{z} \text { (rectangle) }}>K_{\mathrm{z} \text { (diamond) }}>K_{\mathrm{z} \text { (ellipse) }}>K_{\mathrm{z} \text { (circle). When } Q \text { is }}$ less than $570 \mathrm{r} / \mathrm{min}, \quad Q_{\text {(diamond) }}>Q_{\text {(ellipse) }}>Q_{\text {(circle }}>$ $Q_{\text {(rectangle) }}>Q_{\text {(square) }}$. In a word, considering $K_{\mathrm{z}}$ and $Q$ comprehensively, square and triangle micro-pores shaft oil seals have better sealing and lubrication effect under the same working condition.

\subsubsection{The influence of $\boldsymbol{h}_{0}$}

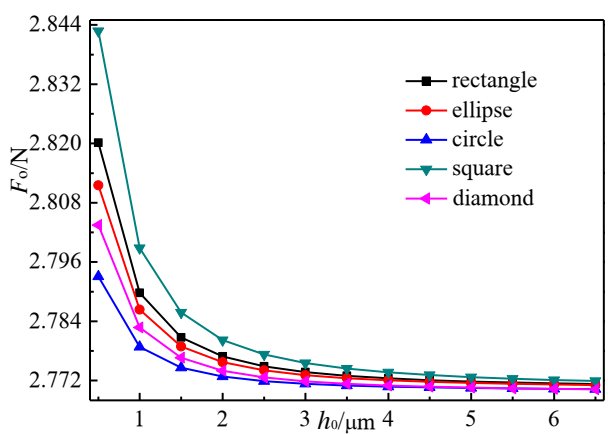

(a) $F_{0} \& h_{0}$

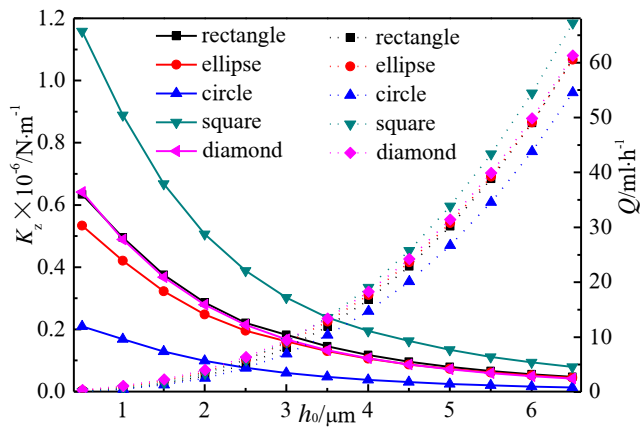

(b) $K_{\mathrm{z}}, Q \& h_{0}$

Fig.5 Effect of $h_{0}$ on sealing performance parameters

The fig. 5 shows the influence law of $h_{0}$ change on the sealing performance parameters in the sealing area. As can be seen from the figure, with the increase of $h_{0}$, the $K_{\mathrm{z}}$ and $F_{\mathrm{o}}$ of each axis surface micro-pores oil seal show a decreasing trend, while $Q$ gradually increases. When $h_{0}$ is smaller, $K_{\mathrm{z}}$ and $F_{\mathrm{o}}$ have a large change range, while $Q$ has a small change range. With the increase of $h_{0}$, the change amplitude of $K_{\mathrm{z}}$ and $F_{\mathrm{o}}$ decreases, and the change amplitude of $Q$ becomes larger. It is indicated that $h_{0}$ has a great influence on the friction lubrication characteristics and sealing performance of the oil seal with micro-pores in the axial surface. Due to the influence of mixed friction, the lubrication and sealing performance of the liquid film will be better when $h_{0}$ is relatively small. When $h_{0}$ is larger, it gradually enters the whole liquid film lubrication state, which results in a gentle decline in the lubrication characteristics and the stability of liquid film, and $Q$ increases. When the $h_{0}$ is the same, it can also be seen that the $K_{\mathrm{z}}$ and $Q$ of each oil seal with micro-pores in the axis surface decrease successively as: square, diamond, ellipse, triangle and circle, and the $F_{o}$ of each oil seal with micro-pores is $F_{o \text { (square) }}>F_{o \text { (rectangle) }}>F_{o \text { (ellipse })}>F_{o \text { (diamond) }}>F_{o \text { (circle) }}$.In conclusion, it is the best choice for better sealing performance when $h_{0}$ is $1.5-4 \mu \mathrm{m}$.

\subsection{Influence of structure parameters on sealing performance}

\subsubsection{Influence of $\gamma$}

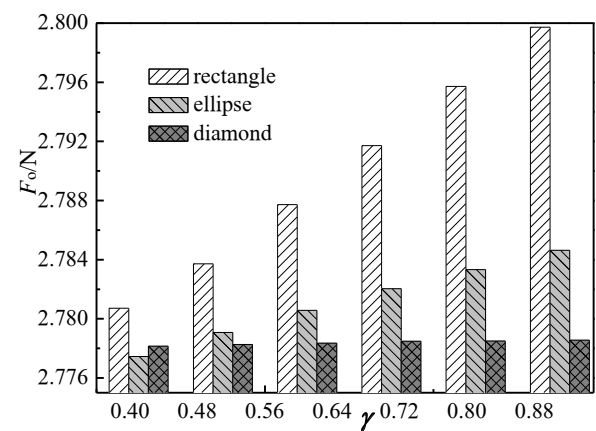

(a) $F_{0} \& \gamma$

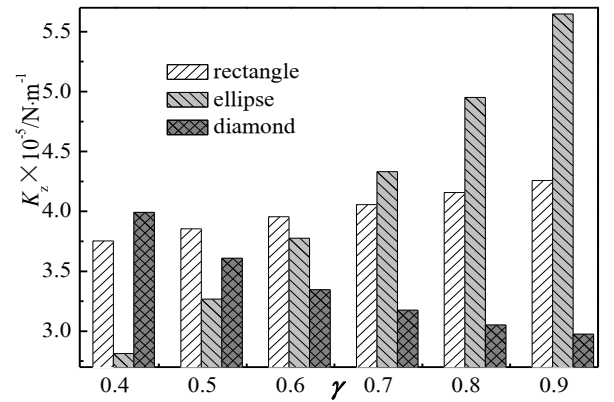

(b) $K_{\mathrm{z}} \& \gamma$

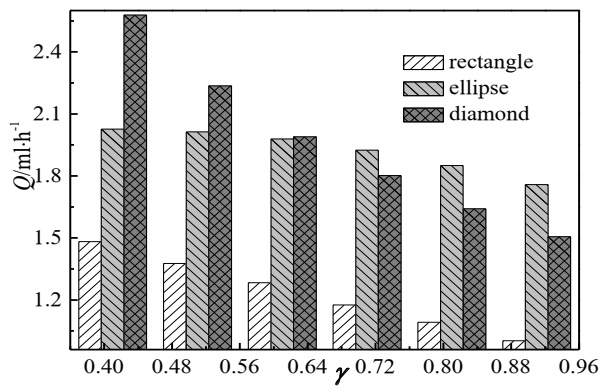

(c) $Q \& \gamma$

Fig.6 Effect of $\gamma$ on sealing performance parameters

It is shown that the influence law of $\gamma$ on the sealing performance of the oil seals in fig.6. With the increase of $\gamma$, the $F_{o}$ of the oil seals with each micro-pore in shaft surface increases gradually, and the $Q$ decreases gradually. $K_{\mathrm{z} \text { (rectangle) }}$ and $K_{\mathrm{z} \text { (ellipse) }}$ increase gradually, and the $K_{\mathrm{z} \text { (diamond) }}$ decrease gradually. When $\gamma$ is the same, it can also see that the $F_{o}$ and $Q$ are ordered as follows: $F_{o \text { (rectangle) }}>F_{o \text { (ellipse) }}>F_{o \text { (diamond) }}$ and $Q_{\text {(rectangle) }}<Q_{\text {(ellipse) }}<Q_{\text {(diamond). }}$ When $\gamma$ is greater than 0.6 , $K_{\mathrm{z} \text { (ellipse) }}>K_{\mathrm{z} \text { (rectangle) })}>K_{\mathrm{z} \text { (diamond) }}$. To sum up, for oil seals, if 
it is required that the liquid film stability and sealing in the sealing area are more reliable, the texture oil seals of the diamond micro-pores when $\gamma$ is $0.5-0.6$ and triangle micro-pores axial when $\gamma$ is $0.8-1.0$ should be selected.

\subsubsection{Influence of $h 1$}

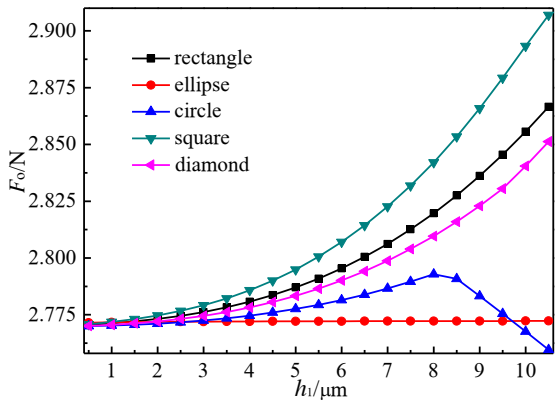

(a) $F_{0} \& h$

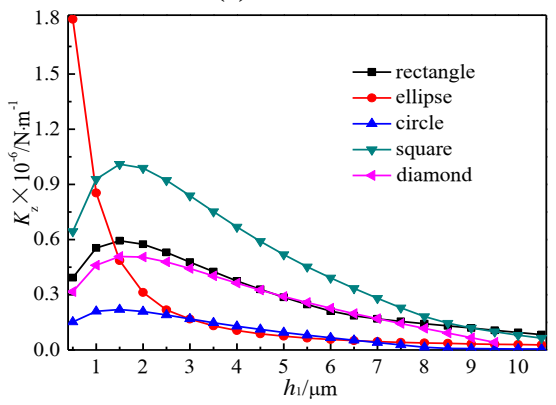

(b) $K_{\mathrm{z}} \& h_{1}$

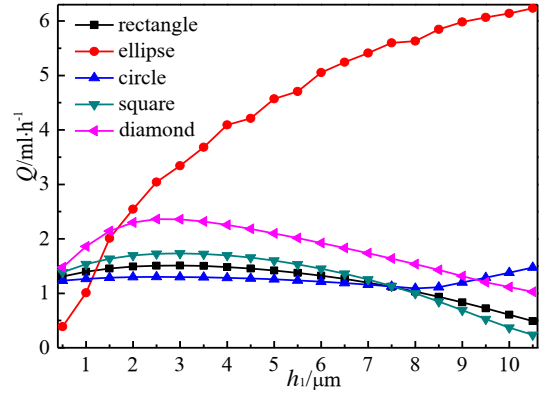

(c) $Q \& h_{1}$

Fig.7 Effect of $h_{1}$ on sealing performance parameters

It is shown that the effect of $h_{1}$ on lip seal performance in the fig.7. Results show that with the increase of $h_{1}$, the open power of other micro-pores shows an increasing trend, except that the open power of circular micro-pores increased first and then decreased. As for the liquid film stiffness and leakage rate, with the increase of pores depth, except that the liquid film stiffness of the elliptic pores oil seal gradually decreases and the leakage rate increases, the liquid film stiffness and leakage rate of axial surface oil seals of other micropores all show a change trend of increasing first and then decreasing. And the liquid film stiffness has a maximum value at $h_{1}=1.5-2.5 \mu \mathrm{m}$, while the leakage rate has a maximum value at $h_{1}=2-4 \mu \mathrm{m}$. With the increase of pores depth, It is suggested that the dynamic pressure effect of the liquid film increases significantly, because of the strong cavitation, shear and extrusion effects of the liquid film in the initial stage, when the pores depth increases to a certain extent, the fluid hydrostatic effect in the micro-pores increases and the squeezing effect decreases, resulting in the reduction of cavitation effect, as a result, the liquid film stiffness and leakage rate increase first and then decrease. Considering the stability of the liquid film in the sealing area and the sealing reliability, the axial surface micro-pores texture interval should be selected when the pores depth is 1.5$4.5 \mu \mathrm{m}$. When the pores depth is the same, it can also be seen that the open power and liquid film stiffness ordering of other micro-pores except for elliptical pores are basically as follows: $F_{o \text { (square) }}>F_{o(\text { rectangle })}>F_{o \text { (diamond) }}>F_{o \text { (circle) }}$ and $K_{\mathrm{z}}$ (square) $>K_{\mathrm{z}}$ (rectangle) $>K_{\mathrm{z}}$ (diamond) $>K_{\mathrm{z}}$ (circle). The order of leakage rate is basically $Q_{\text {(ellipse) }}>Q_{\text {(diamond) }}>Q_{\text {(square) }}>Q$ (rectangle) $>Q_{\text {(circle). In a word, combined with the sealing }}$ performance of each oil seal with micro-pores, the best effect is square, followed by triangle, diamond, circle and ellipse.

\section{Conclusion}

(1) the micro-pores texture in the axial surface can make the liquid film in the sealing area produce significant dynamic pressure effect, which can make the contact surface of the lip seal quickly detach from the axial surface, and enter into the lubrication state of the whole film, so as to avoid contact friction, thus reducing the strict requirements on the roughness and flatness of the axial surface. At the same time, part of the axial surface micro-pores can produce pump drain on oil, the leaking liquid would be back to the oil side, so the sealing reliability can be improved.

(2) With the increase of $p_{0}$, the static pressure effect will be increased which make the $K_{\mathrm{z}}, Q$ and $F_{\mathrm{o}}$ increase, and even cause oil seal failure due to leakage. With the increase of $U$, the bearing capacity and stability of the liquid film were enhanced. When $h_{0}$ increases, the $Q, K_{\mathrm{z}}$ and $F_{\mathrm{o}}$ of oil seal with diamond micro-pores in the shaft surface reduce, and the sealing performance is the best when $h_{0}$ is $1.5-4 \mu \mathrm{m}$.

(3) Considering the stability of the liquid film and sealing reliability in the sealing area, axial texture oil seals with micro-diamond pores of which $h_{1}$ is 1.5 $4.5 \mu \mathrm{m}$ and $\gamma$ is $0.5 \sim 0.6$, or triangular micro-pores of which $\gamma$ is $0.8 \sim 1.0$ should be chosen as the best seal under study condition. The best sealing performance of each micro-pore is square, followed by triangle, diamond, circle and ellipse.

Fund: the National Regional Science foundation of China (No. 51865019); Natural Science Foundation of Jiangxi Province (No. 20201BBE51015)

\section{References}

1. HORVE L A. The correlation of rotary shaft radial lip seal service reliability and pump ability to wear track roughness and micro-asperity formation[R]. SAE paper, 910530, 1991. 
2. BAART P, LUGT P M, PRAKASH B. Review of the lubrication, sealing, and pumping mechanisms in oil and grease-lubricated radial lip seals [J]. J. engineering tribology, 2009, 223(3): 347-358.

3. JIANG Hua-sheng, MENG Xiang-kai, SHEN Mingxue, PENG Xu-dong. Hydrodynamic lubrication performance of lip seal with inclined micro-pores manufactured on rotary shaft surface [J]. CIESC journal, 2015, 66(2): 678-686.

4. GABELLI A, POLL G. Formation of lubricant film in rotary sealing contacts ( I ): Lubricant film modeling [J]. ASME J. tribology, 1992, 114: 280287.

5. PAIGE J, STEPHENS L S. Surface characterization and experimental design for testing of radial lip seal [J]. Tribology trans., 2004, 47(3): 341-355.

6. HADINATA P C, STEPHENS L S. Soft elastohydrodynamic analysis of radial lip seals with deterministic micro-asperities on the shaft [J]. ASME J. tribology, 2007, 129(4): 851-59.

7. WARREN K H, STEPHENS L S. Effect of shaft micro-cavity patterns for flow and friction control on radial lip seal performance a feasibility study [J]. Tribology trans., 2009, 52(6): 731-743.

8. KANAKASABAI V, WARREN K H, STEPHENS L S. Surface analysis of the elastomers in lip seals run against shafts manufactured with micro-cavity patterns [J]. J. engineering tribology, 2010, 224(8): 723-736.
9. JIANG Hua-sheng, PENG Xu-dong, MENG Xiangkai, SHEN Ming-xue. Numerical analysis of reverse pumping rate of rotary lip seal by finite element method[J]. CIESC journal, 2014, 48(8):1194-1199.

10. ZHOU Qiong. Research on the Lubrication Performance of Lip Seal and the Influences on the rotor dynamics performance[D]. East china university of technology, 2013.

11. GUO Fei, JIA Xiao-hong, HUANG Le, WANG Yu-ming. A mixed lubrication theoretical model and experimental verification of rotary lip seals[J]. Journal of mechanical engineering, 2014, 50(3):137-144.

12. SALANT R F. Soft elasto-hydrodynamic analysis of rotary lip seals [J]. J. mechanical engineering science, 2010, 224(12): 2637-2647.

13. Li W,STEPHENS L S,WENK J F. Experimental benchmarking of the numerical model of a radial lip seal with a surface textured shaft[J].Tribology transactions, 2013, 56(1): 75-87.

14. MENG Xiang-kai, BAI Shao-xian, PENG Xu-dong. Lubrication film flow control by oriented dimples for liquid lubricated mechanical seals $[\mathrm{J}]$. Tribology international, 2014(77):132-141.

15. Wen Shi-zhu, Huang Ping. Principles of tribology[M]. Beijing: Tsinghua University Press, 2008: 54. (in Chinese) 